\section{Standard Atomic Weight of Hafnium} Revised
The IUPAC Commission on Isotopic Abundances and Atomic Weights (CIAAW) met under the chairmanship of Dr. Juris Meija (Canada), at the Federal Institute for Materials Research and Testing (bam.de), Berlin Germany, in

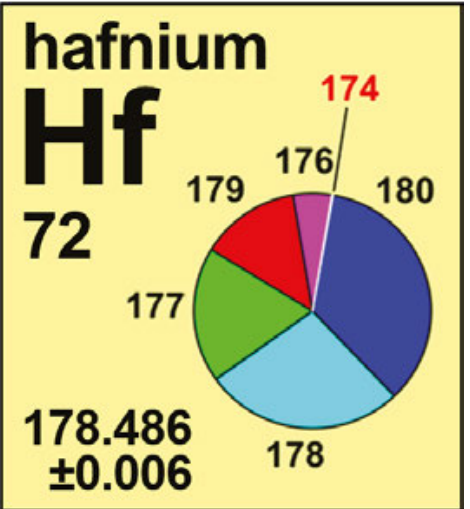

June 2019. As IUPAC celebrates its centennial in 2019, its oldest body, the CIAAW, turns 120 with its beginnings tracing back to Berlin in 1899 [1]. Following its 2019 meeting, the CIAAW recommended changes to the standard atomic weight (i.e. relative atomic mass) of hafnium based on recent determinations and evaluations of its isotopic composition:

hafnium: to $178.486 \pm 0.006$ from $178.49 \pm 0.02$

The CIAAW continues to recognize the extent of natural variations in the atomic weights of the elements. For example, radioactive decay of lutetium alters the isotopic composition of hafnium by producing the light isotope of hafnium-176. Thus, some rare terrestrial materials can have abnormal isotopic compositions of hafnium with the most extreme known case being sedimentary chert from South Africa having atomic-weight value of 178.447.

The CIAAW continues to evaluate literature data measurement science, recognition of new discoveries, and remains committed to modernize its technical guidelines and work towards further expansion of its website to include more historical databases. These changes and considerations will be published in Pure and Applied Chemistry [2] and can be found online at the website of The CIAAW (ciaaw.org).

\section{Notes}

1. For a historical account of the CIAAW, see J.R. De Laeter and J. Meija (23 Jan 2019) "A Weighted Service to Chemistry" IUPAC 100 Stories. Retrieved from https://iupac.org/100/stories/ciaaw-in-the-service-ofchemistry/ and references therein.

2. The "Atomic Weights of the Elements 2017" is to appear next in Pure and Applied Chemistry; see https:// www.ciaaw.org/publications.htm for release. which leads to identification of developments in the

\section{Nomenclature and terminology for}

\section{linear lactic acid-based polymers (IUPAC Recommendations 2019)}

Vert, M., Chen, J., Hellwich, K., et al.

Pure and Applied Chemistry, 2020

Volume 92, Issue 1, pp. 193-211

https://doi.org/10.1515/pac-2017-1007

For more than 60 years, scientists have studied polymers based on lactic acid enantiomers. This family includes aliphatic polyesters and linear, branched, and star copolymers containing lactic acid-based aliphatic polyester segments. These polymers have been developed primarily for applications in biological situations where full performance is required for only a limited period of time, after which degradation and elimination occur. Areas of application include surgery, pharmacology, regenerative medicine, dentistry, ecology, agriculture, packaging, etc. To take full advantage of the outstanding properties of these polymers, it is essential<smiles>C[C@H](O)[C@@H](C)O</smiles><smiles>C[C@H](O)[C@@H](C)O</smiles>

1:1 mixture of $R$ and $S$ enantiomers (racemate)

Preferred IUPAC names (PINs)

(R)-2-hydroxypropanoic acid

(S)-2-hydroxypropanoic acid

rac-2-hydroxypropanoic acid

\section{Other IUPAC approved names}
$(R)$-lactic acid
(S)-lactic acid 
that a unique terminology and mode of naming is used to identify, qualify, and distinguish the members of this polymer family and their particularities.

A polymer can be systematically named using either the 'Structure-based' or the 'Source-based' nomenclature systems extended to non-linear macromolecules. Both systems are general and enable the naming of most organic polymers. However, in the case of chiral aliphatic polymers based on lactic acid enantiomers, academic and patent literatures can be confusing, because specialists as well as non-specialists frequently use different names and abbreviations that do not respect the particularities originating from the source and structure-dependent factors affecting material and degradation properties. In order to facilitate an efficient exchange of accurate information, the IUPAC Subcommittee on Polymer Terminology prepared the present recommendations, which provide the nomenclature and terminology rules and the abbreviations to be respected in all fields concerned with the science and the applications of these degradable polymers.

\section{Brief guide to the nomenclature of organic chemistry (IUPAC Technical Report) \\ Hellwich, K.-H., Hartshorn, R.M., Yerin, A., et al. \\ Pure and Applied Chemistry, 2020 \\ Volume 92, Issue 3, pp. 527-539 \\ https://doi.org/10.1515/pac-2019-0104}

This IUPAC Technical Report is one of a series that seeks to distil the essentials of IUPAC nomenclature recommendations. The present report provides a succinct summary of material presented in the publication Nomenclature of Organic Chemistry-IUPAC Recommendations and Preferred Names 2013, the so-called Blue Book.

The content of this report is also produced as a four-sided lift-out document for easy dissemination (available as PAC supplementary information) and reproduced in this issue as center fold.

See IUPAC project 2010-055-1-800 details and other similar documents, including the Brief Guide to the Nomenclature of Inorganic Chemistry and numerous translations.

\section{https://iupac.org/project/2010-055-1-800}

\section{Nomenclature for boranes and related species (IUPAC Recommendations 2019)}

Beckett, M.A., Brellochs, B., Chizhevsky, I.T., et al. Pure and Applied Chemistry, 2020

Volume 92, Issue 2, pp. 357-383

https://doi.org/10.1515/pac-2018-0205

An appraisal of the current IUPAC recommendations for the nomenclature of boranes and related systems has been undertaken. New developments in the field have been investigated and existing nomenclature systems have been adapted to accommodate these new developments. The principal areas considered are stoichiometric and structural nomenclature (including heteroatom and metal-atom subrogation, as well as substitution of hydrogen), conjoined-cage species, supra-icosahedral systems, and sub-icosahedral non-standard structures. Elements of substitutive, additive, and replacement nomenclature systems have been integrated into individual names to address contentious problems in boron nomenclature that have been around for a long time.

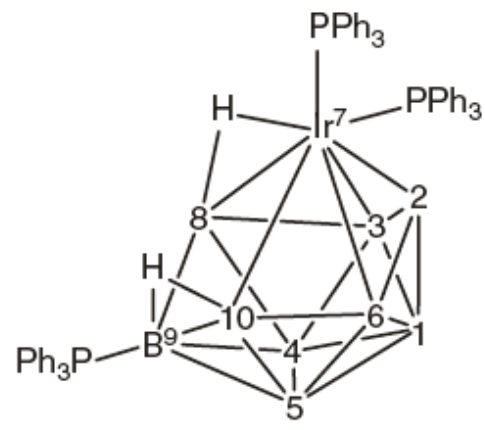

see PAC p. 380 to learn the proper nomenclature of this isonido structure 